\title{
Estrategias de organización y acción política digital
}

Digital organising and political action strategies

José Manuel Sánchez Duarte, Universidad Rey Juan Carlos - josemanuel.sanchez@urjc.es

Raúl Magallón Rosa, Universidad Carlos III - raul.magallon@uc3m.es

Abstract

Political action within networks describes dynamic ways to participate that are constantly redefined. Technology overcomes the formal boundaries of organizations transferring modes, uses and usual practices of political activity (formal and informal) while granting a citizen base and support to act autonomously.

In this article we describe strategies for political action developed within networks from the perspective of organizations for political purposes (from political parties to social movements). The main objective is to offer new methods for an approach to the digital communication policy, mainly in features and trends of digital organization and digital action.

Keywords

Political communication, cyber-activism, cyber-democracy.

\section{Resumen}

La acción política en red describe modos de participar dinámicos y en permanente redefinición. La tecnología supera los límites formales de las organizaciones traspasando los modos, usos y prácticas habituales de la actividad política (formal e informal) a la vez que concede base y soporte a la ciudadanía para actuar de manera autónoma. En el siguiente artículo se exponen los rasgos para el desarrollo de estrategias de acción política impulsadas en la red desde la óptica de las organizaciones con fines políticos (desde partidos a movimientos sociales). El objetivo principal se centra en ofrecer posibles vías de aproximación a la comunicación política profundizando en los rasgos y tendencias de organización y acción digital.

\section{Palabras clave}

Comunicación política, ciberactivismo, ciberdemocracia.

Sumario

1. Rasgos para la elaboración de una estrategia política en red. 2. Organización: De la autonomía informativa a la participación personalizada e intermitente. 2.1. Continuidad y vinculación informativa. 2.2. Pertenencia intermitente y militancias digitales flexibles. 2.3. Hoja de ruta de participación personalizada. 3. Acción para conectar: identidades híbridas, espacios de encuentro y lenguajes en apertura. 3.1. Identidades digitales (híbridas). 3.2. Redes sociales y otros espacios de encuentro. 3.3. Nuevos lenguajes y códigos. Memes e ironía como subcódigos culturales. 4. Conclusiones. 5. Bibliografía. 


\section{Rasgos para la elaboración de una estrategia política en red}

Las organizaciones clásicas de militancia política, definidas tradicionalmente entre afiliados a partidos políticos y militantes en movimientos sociales se enfrentan a su adaptación a un ecosistema cambiante en el que las jerarquías se vuelven menos consistentes, la cadena de mando y autoridad se desplaza de forma más rápida y distribuida y la ejecución de las estrategias adquiere autonomía en consonancia con la propia disposición de la red.

Ante cierta ineficacia política y comunicativa, la ciudadanía adquiere mayor relevancia construyendo propuestas y alternativas en las que, mediante la aplicación de tecnología a su alcance, utilizan internet como sede, inciden en cuestiones concretas, proponen reformas 0 cambios de tipo político (De la Cueva, 2015: 24). La innovación al margen de los actores convencionales (organizaciones empresariales, instituciones públicas, empresas tecnológicas, etc.) y el uso de las tecnologías cívicas (herramientas, plataformas, aplicaciones e infraestructuras digitales impulsadas por organizaciones de la sociedad civil, instituciones y empresas con el objetivo de implicar a la ciudadanía en la toma de decisiones públicas (Sánchez Duarte, Bolaños, Magallón y Anderica, 2015: 200) favorece nuevos modos de participación digital a la vez que plantea nuevos retos para la organizaciones. Instituciones que si bien no se mantienen excluidas del todo de los procesos tienen la tendencia a replicar los modos de hacer habituales frente a la necesidad de protocolos y acciones más flexibles y autónomas.

Desde una visión optimista, las prácticas políticas en la red se ven fortalecidas por el surgimiento de una ciudadanía más autónoma en dos niveles. En términos informativos los sujetos pasivos que consumían la información y propaganda política de manera unilateral pueden diversificar sus fuentes informativas compartiendo perspectivas alternativas, publicando sus opiniones e incluso desafiando discursos oficiales (Loader y Mercea, 2011: 758). El otro nivel implicaría una mayor autonomía de acción -sin dependencias de nodos organizativos centrales- que se ve representada por un aumento en las posibilidades de dar a conocer puntos de vista alternativos, diseñar estrategias de movilización propias o replicando las de otros colectivos.

Estas propuestas adquieren relevancia en términos de velocidad de la movilización, viralidad, permeabilidad y alcance de los problemas así como por la capacidad de enfocar la atención sobre estas cuestiones en el corto plazo (Bennett y Segerberg, 2011: 773) sin fuertes barreras de acceso y salida para la participación y sin grandes inversiones de tiempo y dinero (Boulianne, 2009: 207). La disminución de las restricciones a la participación puede favorecer la inclusión de colectivos menos implicados o autoexcluidos del debate y la toma de decisiones políticas (Christensen y Bengtsson, 2011: 912). Entre las razones que permiten este nuevo escenario destaca la posibilidad de desarrollar nuevos lenguajes, modos y prácticas discursivas y expresivas (memes, recursos irónicos) mejorar la interconexión -como en el caso de las organizaciones de la sociedad civil- y la conciencia de los grupos e individuos a escala global (Carty, 2002: 144).

Desde una óptica negativa se destaca como el aumento de la autonomía en el consumo informativo y la proliferación de diversas formas opinativas implica una disminución de la calidad de la deliberación, el conocimiento y la participación. Incluso se cuestiona de si el diálogo elaborado en la red tiene influencia en las decisiones políticas (por ejemplo, en forma de votos) (Robertson; Vatrapu y Medina, 2010: 29) 0 si por el contrario crea un segundo nivel de militancia sin incidencia política, cuya acción se concentra en la opinión y las acciones estéticas o performativas y que reduce significativa el capital social en términos de redes de confianza, reciprocidad y compromiso (Putnam, 1995).

De igual modo, y vinculado con tres de las objeciones expuestas por Yochai Benkler ${ }^{1}$, la sobrecarga informativa puede conllevar a la fragmentación excesiva de los discursos polarizando las comunidades e influyendo en la calidad de la comunidad política (2015: 276). La denominada «objeción de babel» favorecería la proliferación de ciberguetos, enclaves y comunidades deliberativas muy homogéneas internamente y sin capacidad de establecer diálogos e intercambios con el resto de la red (Sunstein, 2001). Como consecuencia de este escenario, la ciudadanía estaría menos informada, la comunicación política se centraría en exclusiva en los afines (Bennett e lyengar, 2010: 31) y la beligerancia, los ataques y la acción de los trolls acabarían dictando el status quo del mundo online (Lanier, 2011: 87).

La segunda crítica a los efectos democratizadores de internet resalta la concepción errónea de concebirlo como un espacio descentralizado y distribuido al concentrar pocos sitios la mayor parte de las visitas. Incluso en una red abierta se da un alto grado de concentración de la atención en torno a unos pocos sitios líderes (Benkler, 2015: 277). Así, se puede apreciar un número de participantes activos en el proceso de comunicación aunque dejando poco espacio para un diálogo más social y político (losifidis (2011: 626), situado en los márgenes de lo más visitado y la lógica del «me gusta» y que confina a la oscuridad de la red a muchos de los otros aportes que podrían elaborar una esfera pública plural (Sousa, Pinto, Costa e Silva $(2013,10)$.

Por último, internet puede socavar la capacidad de los medios de comunicación tradicionales para presionar y fiscalizar la labor de los gobernantes (Benkler, 2015: 277). Como indica Paolo Mancini pese a que el escrutinio público está más difuminado también puede ser menos eficaz (2013: 56). La multiplicidad de voces puede restar coherencia a los mensajes y eliminar la continuidad de las estrategias requeridas para producir un cambio político (Tilly, 2009) e influir y controlar la agenda política.

Estas tendencias condicionan la creación de una estrategia política digital con el objetivo de potenciar las virtudes y contrarrestar las deficiencias de las actividades políticas e identificar rasgos de la participación política en red. Como se aprecia en la Tabla 1, el diseño de esta estrategia recorrería dos planos, uno centrado en la organización (interna y externa) tanto de partidos políticos como de organizaciones de la sociedad civil. El otro plano se vincularía con la acción -no tanto en términos de planificación y estructuración de actividades- como por su capacidad de configurar las condiciones estructurales para aumentar su eficacia. Como indica Sidney Tarrow citando a Mario Diani, «el principal reto de cualquier movimiento es crear modelos organizativos suficientemente fuertes como para llegar hasta las redes y comunidades de protesta informales que unen a unas personas con otras» (Tarrow, 2012: 232).

\footnotetext{
${ }^{1}$ Las otras dos objeciones expuestas por Benkler hacían referencia al control y el acceso a la información a través de Internet en estados autoritarios y a la brecha digital.
} 
Tabla 1: Rasgos para la elaboración de una estrategia política digital desde la organización y la acción.

\begin{tabular}{|c|c|}
\hline ORGANIZACIÓN & ACCIÓN \\
\hline Continuidad y vinculación informativa & Identidades digitales (híbridas) \\
\hline Pertenencia intermitente y militancias digitales flexibles & Redes sociales y otros espacios de encuentro \\
\hline Hoja de ruta de participación personalizada & Nuevos lenguajes y códigos. Memes e ironía como subcódigos \\
culturales.
\end{tabular}

Fuente: elaboración propia.

\section{Organización: de la autonomía informativa a la participación personalizada e intermitente}

La relación entre el entorno red y las instituciones de participación política está condicionada por la ampliación y el desarrollo de la autonomía como eje de la propuesta organizativa. Una mayor soberanía de los sujetos implicados en estas instituciones se desarrolla en tres niveles distintos pero complementarios entre sí. En el primero, la organización asume la función de proveedora constante de información con el objetivo de mantener una estructura de simpatizantes proclive de ser activada. Esta activación contempla el nuevo carácter intermitente y flexible de la militancia en red (segundo nivel) que establece una agenda personal en base a temas y proyectos específicos más que a grandes bloques ideológicos y partidarios (tercer nivel).

\subsection{Continuidad y vinculación informativa}

Los partidos políticos y los movimientos sociales han enmarcado la comunicación política en un contexto de «campaña permanente». La lógica de la campaña se extiende más allá de los días previos a unas elecciones manteniendo los modos y las prácticas de campaña a lo largo de la legislatura. Así, las organizaciones políticas, y más en el ámbito digital, tienen la obligación de proveer de información constante y actualizada a sus simpatizantes manteniendo un contacto directo y frecuente, informándoles de manera personal, agradeciendo su dedicación y apelando a la participación en cualquier evento o acto. Una constante que se activa con las encuestas digitales en medios de comunicación, los procesos deliberativos que se escenifican fuera de los parlamentos y como mecanismo de respuesta ante «ataques» coordinados política y mediáticamente.

Como indica Bruce Bimber, una buena parte de lo que importa en la participación cívica no es la tecnología de la información en sí misma, sino más bien la información transmitida por ella (2010: 331). En términos estratégicos dotar de información permanente a los simpatizantes puede tener tres utilidades organizativas que traduzcan la información en participación:

- Genera vinculación creando potenciales agentes electorales. Una de las demandas habituales de los cibermilitantes de los partidos políticos era la desatención y la falta de contacto con la organización más allá de los periodos electorales (Sánchez Duarte, 2015). Establecer un canal constante y oficializar vías permanentes de información puede contribuir a la creación de "estructuras de reserva», grupos potenciales en activo que pueden reincorporarse a una reivindicación cuando surgen nuevas crisis y oportunidades (Tarrow, 2012: 245).

- Evita las exclusiones de los grandes medios de comunicación. La creación de un discurso alternativo a enfoques hegemónicos se hace más factible con la universalización de internet. Como contrapartida a la sobreabundancia y la «objeción de babel», la red permite cuestionar los criterios sobre la producción de medios incluida la veracidad, exactitud, exhaustividad, el pluralismo de opiniones, establecimiento de la agenda, las tendencias ideológicas o los modos de dirección (Dahlgren, 2005: 151) Las organizaciones pueden neutralizar, fortalecer o rectificar los discursos de los medios estableciendo una comunicación constante y regular con sus simpatizantes.

- Contribuye a fortalecer una «espiral de la atención selectiva» afín. El consumo de información política se hace por selección, con una fuerte tendencia a consumir aquellas informaciones más afines y a obviar las diferentes (Bennett e lyengar, 2010: 31). La exposición selectiva refuerza la búsqueda activa de información que apoya las propias creencias y evita las fuentes de evitar que las desafían (disonancia cognitiva) (Johnson, Bichard y Zhang, 2009: 74). En el caso de la red, y como se expuso antes, la fragmentación y polarización de las comunidades políticas favorece a la creación de una "espiral de la atención selectiva» (Neuman, Bimber y Hindman, 2010: 24) que refuerza las ideas preconcebidas y que puede ser aprovechado por las organizaciones para fijar discursos.

\subsection{Pertenencia intermitente y militancias digitales flexibles}

En paralelo a la erosión de lealtades partidistas y afiliaciones uniformes, la red presenta un modelo de participación que se vincula con la organizaciones formales de gestión política pero que incluye modos distintos de implicación. La membresía tradicional reducida en muchas ocasiones al pago de una cuota o a la participación en una asamblea se amplía gracias a la red. La auto-organización y coordinación de 
protestas y acciones políticas en ausencia de un grupo de interés y sin coordinadores centrales (Flanagin, Stohl y Bimber, 2006: 30) resultan más factibles y realizables.

Por ello, las formas de militancia que emplean la red como base y soporte de sus acciones (Van Laer y Van Aelst, 2010) exigen diferentes modos de participar menos exclusivos, más flexibles y con varias vías y niveles de implicación. La clave de la participación política en red reside en cuestionar el sentido de la propia militancia. Frente a la heroicidad del afiliado tradicional las militancias actuales tienden a escapar de los excesos de un compromiso invariable y muchas veces acrítico. Así definirían una hoja de ruta personalizada, con intervenciones intermitentes, alejadas de bloques monolíticos: pueden elegir cuándo y cómo contribuir a las organizaciones ganando en independencia y autonomía.

Plantear este debate como un enfrentamiento entre lo viejo y lo nuevo o entre una participación on-line y off-line no resulta lo adecuado. Si bien se ha producido cierta inclusión mínima en las campañas de personas al margen de la política, la colaboración en las organizaciones se debe más a la activación de una militancia en «standby» en referencia a una ciudadanía interesada e informada en la política que se mantiene sin participar pero en un estado de latencia factible de ser activada (Amnå y Ekman, 2014). Ante una oportunidad política y ciertas motivaciones (fuertes liderazgos apartidistas, agravios de otros candidatos) deciden implicarse y actuar.

La acción política en la red favorece la inclusión de estos «militante distanciados» que sustituyen el compromiso total por formas de participar intermitentes (Pudal, 2001: 29) y que combinan sin problemas la participación en partidos políticos convencionales, movimientos sociales y proyectos de innovación ciudadana. Una «promiscuidad política» (Subirats, 2015: 128) que favorece el surgimiento de modos de compromiso e interacción híbridos que contemplan un modelo emprendedor de participación con mayor grado de autonomía, sin la sanción de las autoridades centrales (Flanagin, Stohl y Bruce Bimber, 2006: 34) y con sus propias herramientas, plataformas y dispositivos para el diseño de estrategias y actividades.

\subsection{Hoja de ruta de participación personalizada}

El tercer nivel de autonomía en el marco de la acción política digital complementa los dos niveles anteriores y, en parte, se deriva de ellos. La pertenencia intermitente descrita antes también revela una hoja de ruta propia de organización. Así, las organizaciones deberían enmarcar los problemas no tanto en enfoques macro e inamovibles sino en marcos flexibles, factibles de ser modelados, ampliados y modificados por sus militantes. El marco de desarrollo de esta planificación política flexible se relaciona con la modificación de las normas, los protocolos y los márgenes de lo político, de forma que se hace más abierta y susceptible de nuevos vínculos, al ser capaz negociarse y reconfigurarse (Beck, 2001: 61-62) al asumir la lógica remix de la red.

La posibilidad de realizar una planificación personalizada de la participación va en paralelo al desarrollo de un tipo de política de defensa, causas 0 centrada en problemas específicos. Campañas promovidas por movimientos sociales, partidos políticos y grupos de interés (Dahlgren, 2005: 155) no sólo empresariales sino también configurados como lobbys sociales desde la ciudadanía (Anderica, 2014) que se reinterpretan, con mayor amplitud, rapidez y profundidad, en el entorno digital. Los estilos de vida personales acaban configurando la agenda de preocupaciones de una manera más individual. Como apuntan Lance Bennet y Alexandra Segerberg:

la comunicación personalizada, en el contexto digital, consiste en proporcionar mayores oportunidades a las personas para definir los temas en sus propios términos y en red con otros y a través de los medios de comunicación social (2011: 773)2.

La planificación propia de la militancia -a través o empleando la red como soporte- se interpreta a veces como desapego, desafección política y pérdida de eficacia e incidencia. Sin embargo, la autonomía de información, militancia y organización propia podría ser interpretada como un comportamiento puramente racional en el que la acción se activa en el momento que se necesita (Amnå y Ekman, 2014: 5) y siempre que se dan las bases y el contexto para su materialización de manera efectiva.

\section{Acción para conectar: identidades híbridas, espacios de encuentro y lenguajes en apertura}

Cualquier organización política debe configurar una serie de herramientas con las que materializar la estrategia planificada y desarrollar su ideario. Estos « repertorios de acción colectiva» conforman un «conjunto de medios disponibles para un grupo de personas que los utilizan para actuar de manera colectiva con el fin de obtener objetivos individuales o colectivos» (Tilly, 2009). Con el entorno digital, los repertorios han desarrollado una " acción conectiva» que permite a los ciudadanos activos compartir, fusionar, reproducir y tolerar diferentes identidades políticas mientras el contenido viaja a un ritmo más rápido (Bennett y Segerberg, 2011). Este tipo de acciones complementarias a los repertorios clásicos requieren unas condiciones favorables para su implantación que van desde la construcción de un entorno identitario a partir de un relato común, la existencia de espacios de encuentro (formales e informales) y el desarrollo de lenguajes y narrativas alejadas de las prescripciones clásicas de la política.

\subsection{Identidades digitales (híbridas)}

La participación a través de una acción concreta, la elaboración de un discurso político, implica constituir una identidad colectiva. La creación de un «nosotros» tiene como utilidad identificar y visualizar a un colectivo y a sus acciones (Passerin, 2012: 203). Las identidades reivindicadas son a menudo la indumentaria externa utilizada por las organizaciones para distinguir a sus miembros de los demás (Tarrrow, 2014: 266). También para crear un «afuera constitutivo» en el que el «nosotros» se distingue del «ellos» estableciendo una frontera y

\footnotetext{
${ }^{2}$ Algunos de los ejemplos recogidos por estos autores se centraban en la preocupación por el cambio climático (adoptando una postura en relación a la producción personal de CO2) o en relación al consumo de alimentos (vinculando la acción política a prácticas de comercio justo o a movimientos de «vida lenta»).
} 
dando sentido, por tanto, a la existencia de la propia comunidad (Mouffe, 2014: 295). Las identidades politizadas están así en un continua negociación con el interior y el exterior de sus comunidades y con la necesidad constante de ser reforzada, especialmente tras el pico de una movilización (Tarrow: 2012, 267-268) o acción específica.

En la red, la identidad digital puede definirse como un lazo tecnológico entre una entidad real (persona, organismo 0 empresa) y una entidad virtual (su representación o representaciones digitales) (López, 2015: 482). En el terreno de la acción política dicha identidad puede configurarse y fortalecerse desde ámbitos tanto internos como externos. La elaboración de protocolos de información interna (listas de correos) (Sommerfeldt, 2010: 87) o el empleo de canales de micromensajería instantánea como Telegram o Whatsapp resultan relevantes para mantener una identificación clara y permanente con la organización. En el plano exterior, el uso de plataformas de deliberación y discusión, más allás de las redes sociales, favoreciendo la acción expresiva y convertiéndose en un modo de afirmar, dentro de la esfera pública, los valores del grupo y sus ideales (Dalhgren, 2005: 155).

En el proceso de configuración de las identidades en la red resulta preciso destacar como, frente a enfoques y prácticas políticas convencionales, la lógica digital permite el desarrollo de identidades híbridas. La militancia intermitente y la posibilidad de configurar una hoja de ruta propia de participación, como marco de desarrollo, favorecen la adopción de identidades múltiples y la flexibilidad estratégica (Heaney y Rojas, 2014: 1051) representando una manera de adaptación eficaz a la incertidumbre ambiental (Minkoff, 2002: 382). Las organizaciones deben valorar esas masas híbridas politizadas (con identidades políticas híbridas) (Lasén y Albéniz, 2008: 262) por su potencialidad al valorar la política no como un resultado sino como un proceso alterable, movible, replicable y en una continua remezcla.

\subsection{Redes sociales y otros espacios de encuentro}

En participación política digital las redes sociales ocupan un poder hegemónico. Plataformas destinadas a otras actividades varían su finalidad con el uso que la ciudadanía hace de ellas convirtiéndose en la arena y ámbito central de la ciberpolítica. En la actualidad estas redes se configuran como entornos socio-técnicos que permiten el discurso en la esfera pública (Robertson, Vatrapu y Medina, 2010: 29). De igual modo, refuerzan la idea de autonomía descrita antes potenciando prácticas y acciones personales de los militantes al margen de las organizaciones.

Sin embargo, existe la necesidad de combinar estos espacios con otro tipo de plataformas y recursos digitales al requerir ciertos grupos de espacios separados donde se puedan resolver problemas internos o cultivar una identidad colectiva (Dalhgren, 2005: 153). Este tipo de herramientas podrían destinarse a la creación colectiva de los programas electorales, la discusión de debates sobre actividades y líneas ideológicas del partido, la comunicación estratégica o incluso la formación política de la militancia. En definitiva ir más allá de la mera información y exposición de las redes a la creación de espacios de organización y colaboración. El desarrollo de este tipo de herramientas implicaría tres posibles beneficios:

- Generación de compromiso. Una de las críticas más repetidas a la relación entre internet y las comunidades políticas y sociales es la degradación del capital social. Sin embargo, la creación de espacios propios de pertenencia con pocas barreras de acceso y salida, y no sólo desde las organizaciones sino también desde los afiliados y simpatizantes, podría regenerar y fortalecer dicho capital.

- Fortalecimiento de extensiones virtuales y complementarias: Una de las estrategias de la campaña digital de Barack Obama consistió en la creación de la red social MyBO como espacio para generar rituales compartidos, favorecer intercambios cooperativos e generar identidades colectivas. El objetivo de estos espacios residiría en renunciar a ser mayoritarios para centrarse en redes ya movilizadas, «redes comunitarias» 0 «extensiones virtuales» (Diani, 2000: 396) que precisan combinar estos espacios con encuentros fuera de la red.

- Creación y desarrollo de «redes sedimentarias». La lógica digital implica pensar en acciones políticas fragmentadas y con altos grados de autonomía. Crear espacios propios muy conectados entre sí pero con una ausencia de control centralizado posibilita revivir organizaciones mayores 0 existentes, diseñando sobre la marcha nuevas demandas o estrategias de respuesta. Las «redes sedimentarias» formarían subunidades de acción política (Chadwick, 2007: 294) contribuyendo al desarrollo de estrategias políticas digitales eficaces.

\subsection{Nuevos lenguajes y códigos. Memes e ironía como subcódigos culturales}

En un estudio sobre el perfil de los cibermilitantes en la campaña de las Elecciones Generales de 2008 se identificaba la irrupción de una tendencia de hacer política en la red distanciada de las formas más tradicionales. Los ciberactivistas lúdicos e irónicos convivían con otros perfiles más clásicos ligados a partidos políticos y movimientos sociales basando sus prácticas en red en la realización o distribución de recursos satíricos o parodias sobre la campaña. Si bien en muchos casos no creaban estos contenidos, sí participaban enviando mensajes divertidos y originales sobre temas y propuestas relacionados, sobre todo, con los candidatos (Sampedro, Sánchez Duarte y Campos: 2014) $)^{3}$. Esta tendencia suponía, con ciertos matices, la traslación del infoentretenimiento televisivo a la red. Más en concreto en su fórmula de infosátira, un género surgido en los años 90 y en el que se realizaba un tratamiento paródico y satírico a los asuntos públicos (Valhondo, 2012).

Con el desarrollo tecnológico y la irrupción de las redes sociales como arena predominante del debate político en red, las prácticas lúdicas e irónicas han crecido exponencialmente consolidando nuevos modos de participar y actuar fusionando discursos y políticas subculturales (como los provenientes de la «Cultura Jamming») (Chadwick, 2007: 293). Formas que superan la manera tradicional de concebir la política,

\footnotetext{
${ }^{3}$ Como señala Miguel del Fresno, en la campaña de las Elecciones Generales de 2015 y en el contexto de uno de los debates televisivos entre los principales candidatos, casi un $40 \%$ de la conversación social generada en redes sociales estuvo desvinculada de los partidos y sus equipos de social media y dedicada a generar contenidos que en buena medida estaban orientados al humor, la ironía el sarcasmo ajeno.
} 
asumen planteamientos más performativos y son reivindicadas como actos soberanos de participación (Chouliaraki, 2010). La participación pasa de una formalidad más rígida a integrarse en otro tipo de procesos híbridos donde el ocio y el entretenimiento acaban permeando las prácticas militantes en un marco de acción multitarea en el que se hace política mientras se escucha música, se ven videos en YouTube 0 se contacta con amigos y conocidos.

Los memes, clips irónicos, formatos visuales, audios o diseños gráficos concretan esta tendencia política ya que:

- Se convierten en un producto eficaz, viral y de rápida implantación hasta el punto de dominar la conversación en torno a eventos políticos clásicos como un debate televisivo. Esta característica refuerza su importancia en cualquier estrategia política digital al traspasar fronteras discursivas, institucionales y tecnológicas y crear formas-medios de comunicación híbridas (Baym y Shah 2011: 1034).

- $\quad$ Suponen recursos de auto-expresión para afiliados y militantes a través de la formación de recursos (Loader y Mercea, 2011: 761), no sólo de una manera individual sino también de cooperativo, entre distintos miembros de la organización (Thorson et alt. 2013: 424).

- En esta línea y como señalan Geoffrey Baym y Chirag Shah, estos recursos se plantean como de afinidad para ayudar en el desarrollo de la identidad colectiva y, potencialmente, como recursos deliberativos que pueden ampliar el conjunto de argumentos a favor y en contra de una variedad de posiciones (2011: 1025).

- Al conformar formas de hacer política amplia y menos prescriptiva y emplear códigos y lenguajes más abiertos (como por ejemplo los empleados en las herramientas de mensajería instantánea y en las redes sociales) puede suponer la inclusión de colectivos tradicionalmente excluidos (o autoexcluidos) del debate político.

\section{Conclusiones}

Los ecosistemas políticos, tanto los que podemos considerar más institucionales como los que contemplan canales más alternativos, precisan complementarse con estrategias digitales que abarquen la organización y la acción. Estas estrategias pasan por mantener un contacto directo con los militantes y simpatizantes de las organizaciones a través de una vinculación informativa permanente en la red e identificando nuevos tipos de membresía y participación más flexibles y personalizados. De igual manera, y en términos de acción en red, deberían asimilar actividades y protocolos con los que integrar las nuevas identidades híbridas digitales que se amplían, fortalecen y modulan en espacios digitales y que emplean lenguajes y subcódigos culturales.

Para ello, tanto partidos políticos como movimientos sociales y organizaciones de la sociedad civil deben ampliar las fronteras de la participación más allá de las nociones prescriptivas y convencionales de implicarse hacia una política de la vida cotidiana. De igual modo, debieran asumir que ante escenarios digitales abiertos, flexibles y colaborativos la autonomía de los militantes y la restricción de barreras de entrada y salida a la colaboración resultan claves para el desarrollo de estrategias de acción eficaces. Y todo ello a pesar del coste que puede suponer que la dirección de las organizaciones y los militantes más implicados pierdan el control de la planificación y los mensajes. Este cambio de objetivos, organizativos y de acción, pueden reportar beneficios en el corto y largo plazo al practicar una escucha activa, conectar con los simpatizantes en ambiente menos restrictivos y, tal vez, aumentar su compromiso con la organización.

En definitiva, las estrategias de organización y acción política en red deben evitar su confinamiento en espacios asumidos como naturales para la práctica política (Facebook, Twitter...) combinando herramientas, modos y prácticas. Como indica Byung-Chul Han en el entorno digital «ni los tweets, ni las informaciones se cuentan para dar lugar a una narración. Tampoco la timeline narra ninguna historia de vida, ninguna biografía. Es aditivo y no narrativo» (2014: 60). Las instituciones de gestión política deben establecer un relato digital permitiendo diferentes grados de participación, reconociendo distintos tipos de militancia y posibilitando espacios y protocolos de encuentro.

\section{Bibliografía}

Anderica, V. (2014). Retos comunicativos de la sociedad civil: Del lobby social a la agenda pública. Revista de Estudios de Juventud, 105. pp. 71-90.

Amnå, E., \& Ekman, J. (2014). Standby citizens: diverse faces of political passivity. European Political Science Review, 6(02), pp. 261-281. DOI: http://dx.doi.org/10.1017/S175577391300009X

Beck, U (2002). La sociedad del riesgo global. Madrid: Siglo XXI.

Benkler, Y. (2015) La riqueza de las redes. Como la producción social transforma los mercados y la libertad. Barcelona: Icaria.

Bennett, L. \& lyengar, S. (2010). A New Era of Minimal Effects? The Changing Foundations of Political Communication, Journal of Communication, 58 (4), pp. 707-731 DOI: http://dx.doi.org/10.1111/j.1460-2466.2008.00410

Bennett, L. \& Segerberg, A. (2011). Digital media and the personalization of collective action, Information, Communication \& Society, 14 (6), pp. 770-799. DOI: http://dx.doi.org/10.1080/1369118X.2011.579141

Bimber, B. (2010). The Study of Information Technology and Civic Engagement, Political Communication, 17: pp. 329-333. DOI: http://dx.doi.org/10.1080/10584600050178924

Baym, G. \& Shah, C. (2011). Circulating Struggle. Information, Communication \& Society, 14 (7), pp. 1017-1038. DOI: http://dx.doi.org/10.1080/1369118X.2011.554573 
Boulianne, S. (2009). Does Internet Use Affect Engagement? A Me-ta-Analysis of Research. Political Communication, 26, pp. 193211. DOI: http://dx.doi.org/10.1080/10584600902854363

Carty, V. (2002). Technology and Counter-hegemonic Movements: The case of Nike Corporation, Social Movement Studies, 1:2, pp. 129146. DOI: http://dx.doi.org/10.1080/1474283022000010646

Chadwick, A. (2007). Digital network repertoires and organizational hybridity. Political Communication, 24(3), pp. 283-301. DOI: http://dx.doi.org/10.1080/10584600701471666

Chouliaraki, L. (2010). Self-mediation: New Media and Citizenship. Critical Discourse Studies, 7(4), pp. 227-232. DOI: http://dx.doi.org/10.1080/17405904.2010.511824

Christensen, H. S. \& Bengtsson, A (2011): The political competence of internet participants. Information, Communication \& Society, 14 (6), pp. $896-916$

Dalhgren, P. (2005). The Internet, Public Spheres, and Political Communication: Dispersion and Deliberation, Political Communication, 22, pp. 147-162. DOI: http://dx.doi.org/10.1080/10584600590933160

De la Cueva, J. (2015). Manual del ciberactivista. Teoría y práctica de las acciones micropolíticas. Córdoba: Bandaàparte Editores.

Diani, M. (2000). Social Movements networks. Virtual and Real. Information, Communication \& Society, 3(3), pp. 386-401. DOI: http://dx.doi.org/10.1080/13691180051033333

Diaz Arias, R. (2014). Iconos audiovisuales en las redes sociales: del 15M al 12M. Estudios sobre el Mensaje Periodístico, 20(1), pp. 49-65. DOI: http://dx.doi.org/10.5209/rev_ESMP.2014.v20.n1.45217

Ekman, J., \& Amnå, E. (2012). Political participation and civic engagement: Towards a new typology. Human Affairs, 22(3), pp. 283-300. DOI: $10.2478 / \mathrm{s} 13374-012-0024-1$

Flanagin, A. Stohl, C. \& Bimber, B (2006). Modeling the Structure of Collective Action. Communication Monographs, 73 (1), pp. 29-54. DOI: http://dx.doi.org/10.1080/03637750600557099

Han, B.-C. (2014) En el enjambre. Barcelona: Herder.

Heaney, M. T. \& Rojas, F. (2014). Hybrid Activism: Social Movement Mobilization in a Multimovement Environment. American Journal of Sociology, Vol. 119 (4), pp. 1047-1103. Recuperado de: https://server1.tepper.cmu.edu/Seminars/docs/Rojas_paper.pdf

Holzer, B. \& Sørensen, M. (2003). Rethinking Subpolitics: Beyond the 'Iron Cage' of Modern Politics? Theory Culture \& Society, 20, pp. 79102. DOI: http://dx.doi.org/10.1177/0263276403020002005

Iosifidis, P. (2011). The public sphere, social networks and public service media, Information, Communication \& Society, 14 (5), pp. 619 637. DOI: http://dx.doi.org/10.1080/1369118X.2010.514356

Johnson, T.J., Bichard, S.L. \& Zhang, W. (2009). Communication Communities or "CyberGhettos?": A Path Analysis Model Examining Factors that Explain Selective Exposure to Blogs. Journal of Computer-Mediated Communication, 15 (1), pp. 60-82. DOI: http://dx.doi.org/10.1111/j.1083-6101.2009.01492

Lanier, J. (2011), Contra el rebaño digital. Un manifiesto. Barcelona: Debate.

Lasén, A. y Albeniz, (2008) Movimientos, "mobidas" y móbiles: un análisis de las masas mediatizadas. En Sádaba, I. y Gordo, A. (coords.) Cultura digital y movimientos sociales. Madrid: La Catarata.

Loader, B. \& Mercea, D. (2011). Networking Democracy?. Information, Communication \& Society, 14:6, pp. 757-769. DOI: http://dx.doi.org/10.1080/1369118X.2011.592648

López Gabrielidis, A. (2015). Régimen de visibilidad y vigilancia en la era de la Identidad Digital. Revista Teknokultura, 12(3), pp. 473-499. DOI: http://dx.doi.org/10.5209/rev_TK.2015.v12.n3.50385

Mancini, P. (2013). Media Fragmentation, Party System, and Democracy. The International Journal of Press/Politics, 18(1), pp.44-60. DOI: http://dx.doi.org/10.1177/1940161212458200

Minkoff, D. C. (2002). The emergence of hybrid organizational forms: Combining identity-based service provision and political action. Nonprofit and voluntary sector quarterly, 31(3), pp. 377-401.

Mouffe, C. (2012). Ciudadanía democrática y comunidad política. En Mouffe, C. (Ed.) (2012) Dimensiones de la democracia radical. Pluralismo, ciudadanía y comunidad. Buenos Aires: Prometeo.

Neuman, R.; B. Bimber \& Hindman, M. (2010). Internet and Four Dimensions of Citizenship. The Oxford Handbook of American Public Opinion and the Media. Oxford: Oxford Handbook. 
Passerin, M. (2012) Hannah Arendt y la idea de ciudadanía. En: Mouffe, C. (Ed.) Dimensiones de la democracia radical. Pluralismo, ciudadanía y comunidad. Buenos Aires: Prometeo.

Pudal, B. (2011). Los enfoques teóricos y metodológicos de la militancia. Revista de Sociología, (25).

Putnam, R. (1995). Bowling Alone: America's Declining Social Capital. Journal of Democracy, pp. 65-78

Robertson, S. P., Vatrapu, R. K. \& Medina, R. (2010) Off the wall political discourse: Facebook use in the 2008 U.S. presidential election. Information Polity, 15 (2010), pp 11-31

Sampedro, V., Sánchez-Duarte, J. M. y Campos, E. (2014). Participación ciudadana en las cibercampañas electorales. Debates teóricos y una aproximación tipológica. En Ramón Cotarelo y José Antonio Olmeda (Comps.) La democracia del siglo XXI. Política, medios de comunicación, internet y redes sociales. Madrid: Centro de Estudios Políticos y Constitucionales.

Sánchez-Duarte, J.M. (2015). Participación digital en los partidos políticos. Autonomía y prácticas de militancia en red. Dígitos, Revista de comunicación digital, 1, pp. 59-69.

Sánchez-Duarte, J.M., Bolaños-Huertas, M.V., Magallón-Rosa, R. y Anderica-Caffarena, V. (2015). El papel de las tecnologías cívicas en la redefinición de la esfera pública. Historia y Comunicación Social. Vol 20 (2), pp. 193-209. DOI: http://dx.doi.org/10.5209/rev_HICS.2015.v20.n2.51396

Sierra Infante, S. (2012). Humor y crítica social en la red en el entorno del 15M. Discurso y Sociedad, Vol. 6(3), pp. 611-635. Recuperado de: http://www.dissoc.org/ediciones/v06n03/DS6\%283\%29Sierra.pdf

Sommerfeldt, E. (2010). Activist e-mail action alerts and identification: Rhetorical relationship building strategies in collective action, Public Relations Review, 37 (2011), pp. 87-89. doi:10.1016/j.pubrev.2010.10.003

Sousa, H.; Pinto, M. \& Costa e Silva, E. (2013). Digital public sphere: weaknesses and challenges, en Comunicação e Sociedade, vol. 23, 2013, pp. $9-12$.

DOI: http://dx.doi.org/10.17231/comsoc.23(2013).1610

Subirats, J. (2015). Todo se mueve. Acción colectiva, acción conectiva. Movimientos, partidos e instituciones. Revista Española de Sociología, 24, pp. 123-131. Recuperado de: www.fes-sociologia.com/files/res/24/09.pdf

Sunstein, R. C. (2007). Republic.com. 2.0. Princeton, NJ: Princeton University Press.

Tarrow, S. (2012). El poder en movimiento. Los movimientos sociales, la acción colectiva y la política. Madrid: Alianza Editorial

Thorson, K., Driscoll, K., Ekdale, B., Edgerly, S., Thompson, L. G., Schrock, A.. \& Wells, C. (2013). YouTube, Twitter and the Occupy movement: Connecting content and circulation practices. Information, Communication \& Society, 16(3), pp. 421-451. DOI: http://dx.doi.org/10.1080/1369118X.2012.756051

Tilly, C. (2009). Los movimientos sociales, 1768-2009: Desde sus orígenes a Facebook. Barcelona: Critica.

Valhondo, J. L. (2012) Sátira televisiva y democracia en España. La popularización de la información política a través de la sátira. Barcelona: UOC

Van Laer, J. \& Van Aelst, P. (2010). Internet and social movement action repertoires». Information, Communication \& Society, 13(8), pp. 1146- 1171. DOI: http://dx.doi.org/10.1080/13691181003628307

\section{Cómo citar este artículo en bibliografías - How to cite this article in bibliographies / references:}

SÁNCHEZ-DUARTE, J.M,; MAGALLÓN-ROSA, R.. (2016): "Estrategias de organización y acción política digital”. En Revista de la Asociación Española de Investigación de la Comunicación, vol. 3, número 5, pp. 9-16. 